\title{
Entre détachement et intégration : la topographie des constructions en si et le marquage de la structure informationnelle
}

\author{
Gilles Corminboeuf \\ Universités de Neuchâtel et de Fribourg (Suisse) \\ gilles.corminboeuf@unine.ch
}

\begin{abstract}
On se propose d'étudier le rapport entre la syntaxe et la structure informationnelle, en prenant l'exemple des constructions en $s i^{1}$. Cet essai d'articulation des plans syntaxique et fonctionnel a pour objectif de décrire les correspondances qui existent entre les deux niveaux d'analyse. De notre point de vue, la syntaxe ne marque qu'en pointillé le relief informationnel, mais nous avons sélectionné des exemples qui, pour notre objet d'étude, présentent des indices manifestes ${ }^{2}$. On conviendra avec Touratier [1994 : LVII] que «c'est à partir notamment de la structure syntaxique et c'est dans la structure syntaxique que l'interlocuteur construit la structure sémantico-informative du message. Ceci veut dire que, sans être parallèle à la structure informative, la structure syntaxique est un des éléments qui permet d'appréhender la structure informative ». Les marqueurs formels qui sont à l'origine des divers mécanismes de stratification de l'information seront étudiés à travers la triade \{régime - circonstant - énonciation autonome\}, à savoir les trois statuts syntaxiques que peuvent endosser les éléments si $P$. Cette opposition ternaire, une fois justifiée, sera croisée avec le caractère intégré ou détaché de $s i P$, qui nous paraît jouer un rôle de premier plan dans le marquage de la structure informationnelle.
\end{abstract}

\section{Préalables}

\subsection{Le couple substrat / foyer}

Dans le cadre de cette étude, on se gardera d'évaluer et de rendre compte des nombreuses théories disponibles sur le «marché » qui traitent de la structure informationnelle ${ }^{3}$. On se contentera d'emprunter une partie de l'outillage terminologique utilisé par Nølke [1994: 127sq] et Kronning [1996:45sq] dans le cadre de leurs travaux sur la «stratification énonciative», principalement les notions de substrat et de foyer que l'on adaptera librement. Le foyer sera défini comme un élément qui est sous la portée des opérateurs propositionnels (négation, interrogation, etc.) ou qui entre dans un dispositif focalisant (le clivage, par exemple). Le foyer est la partie de l'énoncé présentée comme incorporant l'information nouvelle et / ou pertinente à un moment donné du discours ${ }^{4}$. Le substrat regroupe les éléments qui sont hors foyer. On parlera de focalisation pour l'opération qui consiste à attribuer à un segment le statut fonctionnel de foyer (noté [+ foyer]). Parallèlement, on assignera aux segments si $P$ le trait [+ substrat] en cas d'opération de substratification marquée par des moyens syntaxiques. Les segments si $P$ typés [substrat] désignent des foyers par défaut et les segments typés [- foyer] des substrats par défaut.

\subsection{Un ou plusieurs si?}

On étudiera aussi bien le si des interrogatives indirectes totales (le si «percontatif») que le si hypothétique. On considérera que le si hypothétique et le $s i$ interrogatif sont deux morphèmes distincts 5 . D'un point de vue distributionnel, le si hypothétique introduit un constituant non nucléaire (un circonstant $\S 3$. infra, ou une «énonciation autonome », § 4.), alors que le si interrogatif introduit un complément nucléaire (un complément régime, $\S 2$.). Du point de vue sémantique, il est manifeste que les deux si ne véhiculent pas le même sens. 


\subsection{Le corpus et le cadre théorique}

Ce travail se fonde sur un corpus issu de sources diverses : la presse écrite, l'oral spontané, télévisuel ou radiophonique, des œuvres littéraires, la base textuelle Frantext, le web, le discours scientifique, etc. L'origine des données est signalée entre crochets après l'exemple.

Le cadre théorique dans lequel s'inscrit ce travail est la macro-syntaxe telle qu'elle est conçue et développée par Berrendonner [2002a; 2002b]. Les notions de «clause» et d'«énonciation» seront définies au $§ 4.1$.

\section{L'élément si $P$ est un complément régime}

Le § 2. présente des constructions dans lesquelles le constituant si $P$ est un complément régime direct. Dans les interrogatives indirectes totales, le verbe recteur enchâsse le constituant si $P$.

\subsection{Le cas non marqué : l'intégration de si $P$}

L'élément si $P$ est intégré à droite du verbe, dans les exemples (1) :

(a) Je me demande $s i$ je ne me trompe pas, et si ce n'est pas cette nuit-là que commença leur liaison. [Léautaud $<$ Frantext]

(b) As-tu vérifié si les pneus sont bien gonflés ? [Matzneff $<$ Frantext]

Dans cette situation, le constituant si $P$, en position de foyer par défaut, peut être typé [- substrat].

Voyons les cas particuliers (2):

(a) <à propos d'un match de football $>$ Ce soir, au moment de passer à table, on saura. Si les Bleus on eu tort de se faire des cheveux blancs. $S i$ la Suisse a eu raison d'y croire. [presse]

(b) C'est le voile du palais qui détermine si une consonne est orale ou nasale. [oral $<$ Duval]

Dans l'exemple (2a), on peut analyser le dégroupage - si l'on se fonde sur l'indice ténu que constitue la ponctuation forte - comme une façon de focaliser à la fois la $\mathrm{P}$ matrice et l'élément si $P^{6}$. Dans $(2 \mathrm{~b})$, le dispositif clivé (c'est $\mathrm{x}$ qui y) opère une "promotion » informationnelle du sujet (le voile du palais), au détriment de si $P$.

\subsection{L'antéposition des interrogatives indirectes totales}

Le déplacement en tête des interrogatives en $s i$ est un procédé rarement exploité en français :

(a) Retours, détail des fortunes, description des lieux et des costumes; le lecteur n'est ni ménagé, ni flatté, ni rassuré ; $s$ 'il est content ou non, nul ne s'en soucie ; [Alain $<$ Frantext]

(b) Si le coup d'Etat que voici finira par faire de Hitler le véritable maître de l'Allemagne, cela dépendra du chef raciste lui-même [Dominique $<$ Renchon]

(c) $S i$ cela est vrai ou non, je n'ai pas à le voir ici, mais je ne le veux pas mettre en oubli. $[$ Hugo $<$ Frantext]

Dans (3a), la disjonction (ou non) et dans (3b) la forme verbale au futur de l'indicatif suggèrent la nature interrogative de la $\mathrm{P}$ en si. L'exemple (3c) montre clairement que c'est le verbe recteur (ai à le voir) qui est sous la portée de la négation et non l'élément si $P$. En version détachée - et ce sera aussi le cas pour la postposition, § 2.3 - l'effet est celui d'une substratification (cf. Corminboeuf, à paraître, a).

\subsection{La postposition des interrogatives indirectes totales}

Considérons les cas de postposition des régimes en $s i$ :

(a) Fais-le savoir, si tu viens. [ $<$ Delaveau] 
(b) On me faisait toujours la même réponse. Toujours. Je devais bien le savoir, si j'avais un pansement à la main quand je ne l'avais pas. Je devais bien le savoir, si j'avais dormi dans un hôtel où je n'étais jamais entrée. Je devais bien le savoir, si j'avais téléphoné pour demander le numéro de quelqu'un chez qui, justement, je me trouvais. Tout le monde était sincère. $\S$ Moi j'étais dingue. [Japrisot $<$ Frantext]

Les observables (4) présentent, comme les exemples (3), un phénomène de « double marquage »: le régime introduit par si est, au moyen d'un pronom clitique, cataphorisé dans (4), alors qu'il était anaphorisé dans (3). Les si $P$ interrogatives détachées - à gauche dans (3) ou à droite dans (4) - sont typées [+ substrat]. Dans le cas des interrogatives indirectes totales, le détachement est par conséquent un moyen de substratifier si $P$.

\section{$3 \quad$ L'élément si $P$ est un circonstant}

Le $\S 3$. détaille le comportement des constituants si $P$ qui ont un statut syntaxique de circonstant.

\subsection{Le circonstant si $P$ est hors prédication}

Dans ce paragraphe, la suite de discours introduite par si est un circonstant extra-prédicatif. L'élément si $P$ est détaché, mais néanmoins régi : si $P$ implique syntaxiquement la présence de $\mathrm{Q}^{7}$.

\subsubsection{Le cas non marqué : l'antéposition de si $P$}

La grande majorité des si $P$ circonstancielles sont antéposées :

(a) $[. .$.$] ; et, si j'aime être chatouillé, j'aime pouvoir ne plus l'être dès que ma dignité$ l'exige. [Koltès, Dans la solitude des champs de coton]

(b) $\mathrm{Si}$ cet ouvrage n'a pas été retiré dans les délais, il sera remis en circulation pour d'autres lecteurs. [Circulaire de la bibliothèque]

(c) - On est des salopes de se moquer des profs.

- Ouais, mais s'ils étaient normaux, peut-être qu'on se moquerait pas. [conversation rapportée dans la presse]

On n'entrera pas dans le détail des différences formelles et sémantiques que manifestent les si $P$ dites « factuelles » (5a) par rapport aux si $P$ hypothétiques (5b)-(c). Signalons simplement leur comportement par rapport au clivage : (5a) résiste au clivage, ce qui, à notre sens, confirme son statut a-focal (= hors foyer). Il existe donc des classes de circonstants en si qui ne peuvent jamais être focalisées. Néanmoins, le caractère régi de si $P$ dans (5a) ne fait aucun doute : si $P$ implique syntaxiquement la présence de $\mathrm{Q}$ (cf. infra $\S 4$. pour l'analyse d'éléments si $P$ non régis). En revanche, les circonstants si $P$ de $(5 b)-(c)$ sont clivables et intégrables à droite du verbe ou postposables en position détachée comme (6) infra. Au plan syntaxique, tous les exemples (5) sont pour nous des constituants si $P$ régis par la construction verbale subséquente.

Les circonstants en si de (5) placés en position frontale ont un caractère a-focal que l'on notera [- foyer].

\subsubsection{La postposition non focalisante}

Dans (6), le circonstant si P postposé (si vous êtes passionné) est affecté d'une intonation de postfixe ${ }^{8}$ :

je vous dis quand vous êtes passionné / euh : vous calculez pas euh : pfff $\backslash$ il peut vous arriver n'importe quoi $\backslash$ c'est clair que y a des moments durs mais... la passion $p f f f .$. vous êtes capable de faire n'importe quoi $\backslash s i$ vous êtes passionné_ [oral tv] 
L'objet-de-discours <passion> a fait l'objet d'une validation préalable dans la mémoire discursive, et l'élément si $P$, non intégré intonativement, joue le rôle d'une simple annexe. Ce procédé réparateur a une fonction d'éclaircissement d'un élément probablement perçu comme trop peu saillant cognitivement.

Ce serait donc opérer une généralisation outrancière que de dire que les structures $Q$ si $P$ sont toutes des hypothétiques focalisées (cf. § 3.2.) : suite à une rupture prosodique, le terme si $P$ peut être dé-focalisé à droite $^{9}$. Comme pour les exemples (5), le constituant si $P$ de (6) endosse le trait [- foyer].

\subsection{Le circonstant si $P$ est intra-prédicatif}

\subsubsection{L'intégration à droite}

Il n'est pas anodin de considérer que le circonstant si $P$ puisse occuper une zone focale, puisqu'on tient généralement pour acquis depuis Haiman [1978] que «les conditionnelles sont des topiques ». Dans les exemples (7) et suivants, l'élément si $P$ implique syntaxiquement la présence de $\mathrm{Q}(Q$ régit si $P)$. En revanche, d'un point de vue informationnel la situation s'inverse : une contrainte d'informativité rend l'élément si $P$ inéluctable. Un élément placé dans le champ des modalités comme la négation ou l'interrogation ne bénéficie pas d'une «promotion» syntaxique. Dans nos exemples ci-dessous, ce n'est pas parce que si $P$ renferme le foyer que son statut syntaxique d'élément régi est remis en question. On différenciera par conséquent le marquage de la modalité du statut syntaxique.

Considérons les exemples suivants :

(a) <à propos d'alpinisme > l'engagement personnel est total / car l'avion ne viendra me rechercher que dans trois semaines / et seulement si les conditions météo le permettront $\backslash$ [oral tv]

(b) Je ne m'endormirai que si d'autres s'éveillent. [Eluard, Pouvoir tout dire]

(c) - Mais enfin, tu sais bien que je ne peux pas aller raconter des histoires pareilles à des cognes, quoi ! On serait tous dans le coup.

- Si tu racontes tout, oui, mais pas si tu te contentes de les avertir.

- Mais tu les connais pas [...] [Clavel $<$ Frantext]

La restriction (seulement si / que si) et la négation sont des opérateurs qui ont le foyer sous leur portée. Toute la partie si $P$ fait partie de l'opérande. Dans (7a), l'ellipse de Q après et renforce le statut focal attribué au terme si $P$ : on n'ellipse pas les foyers. Dans (7b), la restriction (que) porte clairement sur si $P$. Le dialogue rapporté en (7c) propose, dans la seconde intervention, un contraste de modalités qui voit se succéder deux foyers antagonistes articulés par le connecteur mais. Dans ces exemples (7), il ne fait aucun doute que si $P$ exprime un foyer.

Les dispositifs clivé et pseudo-clivé entrent également dans ces cas de figure où si $P$ est le foyer :

(a) C'est surtout si les Etats-Unis ne sont pas représentés à Genève qu'ils prennent des risques. [Mendès-France $<$ Frantext]

(b) là où ç'aurait été intéressant c'est s'ils nous avaient dit au début qu'il fallait lire le journal en continu [oral $<$ Roubaud]

Dans la construction clivée (8), si $P$ occupe un emplacement dévolu au foyer; l'adverbe paradigmatisant «surtout » fonctionne également comme un marqueur de focalisation. Pour ( $8 b$ ), le critère de Delaveau [1980] selon lequel le dispositif pseudo-clivé n'est compatible qu'avec les interrogatives indirectes et le clivage qu'avec les circonstants semble ne pas fonctionner ici. Dans (8), on peut faire alterner $s i$ avec un autre circonstant, par exemple au cas où. En revanche, l'insertion d'une disjonction (ou pas) - une propriété associable aux interrogatives - donne un résultat bizarre : ?là où ç'aurait été intéressant c'est s'ils nous avaient dit au début qu'il fallait lire le journal en continu ou pas. A notre sens, l'élément si $P$ de (8b) est donc à analyser comme un circonstant focalisé.

Les comparatives du genre (9) sont à placer également dans le lot des emplois intra-prédicatifs : 
(a) $[\ldots]$ et je n'ai pas fait cent pas que je suis plus brisé que si j'avais fait dix lieues. [Montesquieu, Lettres persanes]

(a') [...] et je n'ai pas fait cent pas que je suis plus brisé que $<$ je ne serais brisé $>$ si j'avais fait dix lieues.

Le régissant $<j e$ ne serais brisé> de (9a') est hautement prévisible, ce qui fait qu'on peut en faire l'économie sans risque de carence d'informativité. Le constituant régi si $P$ se voit ainsi informationnellement promu.

Seuls les éléments récupérables sont ellipsables, ce que démontre (10) de façon spectaculaire :

Philippe, irrité, répliqua : Si j'entre dans la Laconie, je vous en chasserai tous. Ils lui répondirent : $\mathrm{Si}$. [Barthélémy $<$ Littré]

Dans (10), tout est ellipsé sauf le connecteur qui, du coup, porte forcément le foyer. La réponse (Si) se comprend au sens de <encore faut-il que tu entres dans la Laconie>. La structure réactive opère un déplacement du foyer sur l'élément $s i$; le constituant si $P$ dans la première réplique (Si j'entre dans la Laconie, je vous en chasserai tous) était en effet extra-prédicatif.

Une note sur la prosodie :

Dans l'exemple (7), l'élément si $P$ apparaît sous intonème conclusif, en position finale de foyer par défaut ${ }^{10}$. La prosodie corroborerait ici l'hypothèse syntaxique.

La prosodie peut opérer également un marquage du foyer au moyen d'un accent d'intensité sur $s i$ :

On ira au cinéma si on ne va pas à la plage. [ $<$ de Cornulier]

Dans cet exemple signalé par de Cornulier [1985 : 79], si s'interprète comme un ou exclusif.

\subsubsection{Le constituant si $P$ est détaché et focalisé}

$\mathrm{Ce}$ cas de figure, très rare en français, met une fois de plus un doute sur la position affichée par Haiman :

(a) Adopte donc nos coupures. Seulement si nous avons laissé des répétitions, corrige-les.

[Flaubert $<$ Frantext]

(b) Si et seulement si Frank est complètement mis hors de cause par la justice, nous le contacterons pour entendre ses explications sur ce qui s'est passé la semaine dernière, a expliqué Dennis Dejonckere, le porte-parole de Domo. [presse]

Non seulement la «conditionnelle» des exemples (12) n'est pas un «topique» (cf. la portée de l'opérateur de restriction), mais en plus elle est placée en tête de la structure. Le constituant si $P$ est donc focalisable en position frontale : dans (12), l'opérateur de restriction seulement porte clairement sur si $P$. L'effet produit est surprenant, mais il semble que la procédure soit possible, moyennant à l'oral, une prosodie adaptée ${ }^{11}$. Le détachement semble servir ici à focaliser et non à substratifier ! Ce genre de faits empiriques est gênant pour la doxa sur la structuration informationnelle des énoncés qui tend à traiter automatiquement les éléments détachés à gauche comme des substrats. Dans (12), on pourrait considérer que si $P$ n'est pas antéposé, mais que c'est Q qui est postposé (avec une intonation d'« annexe »à l'oral). Cette hypothèse permettrait de ramener ces exemples aux si $P$ intra-prédicatifs, en particulier ceux qui sont sous la portée de la négation et des marqueurs restrictifs (ex. 7).

Les exemples (7) à (12) manifestent des stratégies diverses visant toutes à typer l'élément si $P$ comme ayant le trait [+ foyer].

\subsection{La position de si $P$ est-elle fonction d'autres facteurs que la stratification informationnelle?}

Voyons un exemple des effets de sens imputables à l'insertion du terme si $P$ à l'intérieur du prédicat : 
(a) La branche armée du Hamas a menacé dimanche <si l'offensive militaire se poursuit de frapper en Israël>. [teletext]

(a') Si l'offensive militaire se poursuit, la branche armée du Hamas a menacé dimanche $<$ de frapper en Israël>. [exemple modifié à partir de 13a]

Dans (13a), la construction si $P, Q$ correspond à $y$ dans le prédicat menacer $(y, x): x$ menace $[\text { si } P, Q]_{y}$. La menace est effective que $P$ soit validé ou non. Pour le cas de figure modifié (13a') en revanche, le cadre fictif est sorti de l'opérande, ce qui fait que la menace n'est valide que dans le cadre fictif ouvert par si (si $P, x$ menace $y)^{12}$. La position de si $P$ - à l'intérieur $v s$ à l'extérieur du prédicat - permet de modifier sensiblement le sens. Le distinguo (13a) vs (13a') concerne, selon nous, tout autant le domaine de validité du cadre fictif par rapport au procès que le statut informationnel de si $P$ proprement dit.

Les exemples (14) conduisent aux mêmes conclusions que (13) :

(a) L'ancien ministre a dénoncé «l'entêtement d'un homme, le Premier ministre, qui menace de démissionner si on abroge le CPE ». [teletext]

(b) Le président iranien a à nouveau contesté la légitimité de l'Etat d'Israël. Il a proposé que l'Allemagne et l'Autriche l'accueillent sur leur territoire s'ils s'estiment coupables de massacres de juifs pendant la $2^{\text {ème }}$ guerre mondiale. [web]

Là non plus, il semble que la linéarisation ne soit pas fatalement liée à des impératifs de stratification du prédicat. Dans (14), il est compliqué de réaliser une antéposition du membre si $P$ en raison des propriétés d'enchâssement qui sont ici en jeu [Hamon, 2002]. En effet, si $P$ est compris dans l'enchâssement, c'està-dire qu'il porte sur un élément qui est à l'intérieur de la que-P. C'est selon nous cette contrainte qui explique pourquoi l'antéposition est bloquée, ou du moins rendue difficile. Les exemples (14) présentent un segment en si qui fait partie d'un discours rapporté, ce qui rend peu praticable une antéposition en dehors de l'enchâssement. Pour l'anglais, Ford \& Thompson [1986] notent également que le constituant if $P$ est souvent positionné à droite lorsque qu'il est enchâssé; les auteurs soulignent par ailleurs que le complément if $P$ a tendance à être placé à droite s'il est plus long que son régissant $\mathrm{Q}$ (cf. 14b, supra). Dans ce cas de figure, les contraintes liées à l'organisation informationnelle entrent moins en ligne de compte.

En conclusion de ce paragraphe sur les si $P$ circonstants, on peut dire que dans le cas non marqué, les constituants si $P$ antéposés ne sont pas des foyers (ex. 5), alors que les éléments si $P$ intégrés à droite sous la portée des modalités d'énoncé expriment des foyers (ex. 7). Pourtant, il convient de rester prudent, puisque les cas de dé-focalisation à droite (ex. 6) et de focalisation en position frontale (ex. 12) sont attestés (même si ce sont des cas marqués).

Dans les $\S 2$. et $\S 3$., on a étudié des constituants si $P$ régis par la construction verbale de $Q$ : des compléments « internes » (les régimes) et des compléments « externes» (les circonstants). Dans ces cas de figure, le régi si $P$ implique syntaxiquement la présence du régissant $\mathrm{Q}$. Le $\S 4$. présente une classe de faits où la relation entre si $P$ et $\mathrm{Q}$ ne peut pas être saisie en termes de rection.

\section{L'élément si $P$ est une énonciation autonome}

Les emplois traités ici sont parfois étiquetés «si austinien » parce qu'ils ont été étudiés par Austin [1961 : 210-213].

L'auteur signalait des suites comme (15):

(15) (a) There are biscuits on the sideboard if you want them $[<$ Austin]

(b) I paid you back yesterday, if you remember [ibid.] 


\subsection{L'hypothèse d'une ellipse}

On dira du constituant si $P$ dans des exemples comme Si tu as soif, il y a de la bière dans le frigo qu'il forme une énonciation autonome privée de son régissant; cette énonciation commente l'énonciation qui suit (si $P$ n'est pas un complément régi par Q $)^{13}$. L'énonciation est définie comme l'actualisation en discours d'une clause (avec un schème prosodique, une instance de prise en charge, etc.). Une énonciation est un opérateur sur la mémoire discursive : elle modifie l'état des connaissances partagées par les interlocuteurs. Une clause est une unité qui se définit par la connexité rectionnelle qui existe entre ses constituants $^{14}$; elle est syntaxiquement autonome vis-à-vis de l'environnement. Autrement dit, la clause s'arrête là où s'arrête la rection; le phénomène de rection regroupe essentiellement les implications d'occurrence entre des segments signifiants [Berrendonner, 2002a ; 2002b].

Il n'y a pas de propriétés qui attesteraient que si $P$ entre dans un système de rection (cf. par exemple les tests de rection de Blanche-Benveniste, 1981) :

- $\quad$ on ne peut pas faire porter d'opérateur de restriction ou de négation sur si P : ?Il n'y a de la bière dans le frigo que si tu as soif;

- $\quad$ si P réagit négativement au clivage : ?c'est si tu as soif qu'il y a de la bière dans le frigo ;

- $\quad$ on ne peut pas aisément faire porter un adverbe paradigmatisant sur si P : ?Principalement si tu as soif, il y a de la bière dans le frigo ;

- $\quad$ si $P$ ne peut pas faire l'objet d'un contraste de modalités : *pas si tu as soif, il y a de la bière dans le frigo, mais si tu veux en offrir à Pierre;

- $\quad$ on ne peut pas réaliser une reprise en et cela : *il y a de la bière dans le frigo et cela si tu as soif;

- la suite si $P Q$ entre difficilement dans le champ d'une modalité interrogative : ?Est-ce que si tu as soif il y a de la bière dans le frigo?

En conclusion, on dira que le terme si $P$ implique la présence de $\mathrm{Q}$ au niveau pragmatique uniquement.

Dans notre terminologie, les configurations rencontrées sont des périodes binaires où le terme si $P$ est une énonciation méta-discursive, véhicule d'une instruction cataphorique ${ }^{15}$. Une glose possible serait : si tu as soif <je t'informe de ce qui suit> il y a de la bière dans le frigo : l'élément entre parenthèses angulaires est d'ordinaire ellipsé. Dans des constructions de ce genre, il semble que l'omission du régissant soit rendue possible par le présence du régi. Étant donné le régi, le calcul du prédicat ellipsé consiste ici dans le rétablissement d'un verbum dicendi. Le constituant ellipsé peut s'interpréter de deux manières différentes au moins : soit il renvoie à la totalité de l'énonciation $\mathrm{Q}$, soit à la vérité de l'énonciation actualisée dans $\mathrm{Q}^{16}$. L'élément zéro de l'énonciation méta-énonciative - par exemple <j'énonce ce qui suit $>$ ou $<$ ce qui suit est vrai $>$ - précise la fonction communicative de Q. Pour les faits regroupés dans ce paragraphe 4., l'élément si $P$ marque un acte d'ouverture d'un univers fictif dans lequel s'insère l'acte d'énonciation qui suit (et non un contenu sémantique, comme c'était le cas pour les circonstants).

Les exemples (16) font figure de rareté :

(a) Si vous payez l'impôt sur le revenu comme un Français sur deux ce qui suit vous concerne... Des déclarations d'un nouveau type vont être utilisées dès le printemps prochain... [Texte du TJ sur www.france2.fr, 07.12.2005]

(b) Si nous supposons que le corps humain fonctionne sans heurts, et qu'il en va de même du corps social, alors, je vous le demande : Que ferions-nous de notre temps? [Miller, Lire aux cabinets]

En introduction à ce paragraphe 4.1, il a été précisé que le régissant de si $P$ est ellipsé. Les cas de figure reproduits en (16) sont particulièrement importants pour notre hypothèse puisque, exceptionnellement, l'élément zéro est verbalisé. Le régissant de si $P$ est souligné en italique dans (16). Son caractère métaénonciatif et cataphorique est ici parfaitement explicite. Il s'agit de notre argument central pour attester 
un fonctionnement d'énonciation autonome. L'ellipse est parfois remplie, l'élément zéro alternant avec un verbe de parole.

Les éléments si $P$ macro-syntaxiques sont particulièrement mobiles, ce que démontrent les $§$ 4.2. à 4.4.

\subsection{L'antéposition}

\subsubsection{La portée sur l'énonciation}

Les énonciations méta-discursives de (17) sont paraphrasables par si $P,<j$ 'énonce ce qui suit $>, Q$ :

(a) $s i$ vous voulez voir / ils sont en train de charger le taureau $\backslash$ [oral tv]

(b) $\mathrm{Si}$ vous voyagez en voiture, un car-ferry relie Penedo et Passagem, à courte distance de Neópolis. [guide touristique]

(c) $S i$ vous prenez L'homme au gilet rouge, le rapport entre la tête et le bras qui a l'air tellement long pour les gens, est plus proche des rapports dans une peinture byzantine que dans toute la peinture depuis la Renaissance. [Giacometti, Ecrits]

L'énonciation si P esquisse le cadre dans lequel l'assertion $Q$ est maximalement pertinente.

\subsubsection{La portée sur la vérité du dire}

Dans les exemples (18), l'élément zéro porte sur la vérité du dire :

(a) Si je comprends bien, l'aéroport de Zermatt c'est Genève, et les montagnes de Genève c'est Zermatt? [presse]

(b) Oh ! cela ne tire à aucune conséquence, dis-je, hésitante. Cependant, si on ne doit rien omettre de la vérité, Mr Colemen s'est vanté un jour devant moi de ses aptitudes à imiter des documents aussi bien qu'un faussaire de profession. [A. Christie $<$ Rocq-Migette]

Une explicitation possible pour ce genre de structures prendrait la forme : si $P,<$ ce qui suit est vrai $>, Q$, où l'élément inséré entre parenthèses angulaires est ellipsé.

\subsection{La postposition}

Les énonciations si $P$ peuvent être postposées, comme dans les exemples d'Austin reproduits en (15):

(a) Je suis au bureau 305, si t'as pas de clé. [billet affiché sur une porte]

(b) mais il faut que nous ayons ce vote d'entrée en matière / si on reprend des termes parlementaires $\backslash$ [radio, débat sur l'adhésion à l'UE]

(c) L'enjeu, un demi-manuscrit qui devrait mener à un fabuleux trésor : rien de moins que la mythique Libereia, la bibliothèque des empereurs de Byzance dont la trace s'est perdue à Moscou à l'époque d'Ivan le Terrible. Cette bibliothèque, au fait, qu'est-elle devenue, si elle a jamais existé ? [presse]

Dans (19b), l'élément zéro porte sur le dire en tant que choix du signifiant. Dans (19c), l'élément ellipsé porte sur le réajustement de présupposés, qui sont remis en jeu, voire niés; à noter en effet les anaphoriques la bibliothèque... cette bibliothèque, puis la fragilisation du présupposé <il existe une bibliothèque des empereurs de Byzance>, par l'intermédiaire de l'élément si $P$.

\subsection{L'incidence}

Les énonciations en $s i$ apparaissent sans autre en position d'incidente :

(20) (a) Cela revient à dire - si l'on nous pardonne ce barbarisme - que la prosodie exercerait de manière privilégiée une fonction de type « incidentiel » [...] [discours scientifique] 
(b) Je vous prie de me savoir, Maître et, si vous le permettez, ami $\S$ très respectueusement vôtre [Correspondance Paulhan-Gide]

Dans (20a), le démonstratif ce est cataphorique : les guillemets indiquent que la portée de si $P$ est locale. Dans (20b), une interprétation possible est de considérer que si $P$ porte sur le coordonnant et : si $P$ fonctionne comme un excuse au rapprochement de maître et ami.

Les éléments si $P$ typés «énonciations » sont toujours détachés et sont plus mobiles que les compléments (régimes et circonstants). On trouve par exemple nettement plus de si $P$ macro-syntaxiques en position incidente que de si $P$ 《 régime » ou « circonstant». Quelle que soit la position de l'énonciation si $P$, sa valeur est celle d'un substrat. La position de si $P$ dans les énonciations autonomes ne semble en effet pas liée à des effets de structuration informationnelle, contrairement aux régimes et aux circonstants. De type [- foyer], elles ouvrent un cadre de pertinence pour l'énonciation qui leur est apposée ${ }^{17}$.

\title{
5 Métanalyses
}

Le concept de métanalyse (emprunté à Jespersen puis Blinkenberg [1950:43]) désigne le fait que, pour une même structure, deux analyses syntaxiques concurrentes et équiprobables soient envisageables. La différence de sens est cependant si ténue qu'elle n'est pas significative dans la communication. La notion est importante dans la mesure où elle permet de donner une explication à des phénomènes d'ambiguïté. Certaines constructions neutralisent en effet les oppositions régime - circonstant et circonstant énonciation autonome.

\subsection{Métanalyse régime - circonstant}

Les exemples (21) sont analysables de deux façons :

(a) S'il rêve, il ne s'en souvient jamais. [< Delaveau]

(b) $\mathrm{Si}$ la Lorpailleur est folle, je n'y peux rien. [Pinget, Le libera]

Dans (21), le constituant si $P$ est-il un circonstant ou un régime ? Ces constructions sont en fait parfaitement ambiguës. Pour (21a), dans l'interprétation « circonstant », l'anaphorique en reprend qu'il rêve, alors que dans l'interprétation « régime », il reprend l'ensemble de la disjonction s'il rêve ou pas. Pour (21b) sont possibles une interprétation circonstancielle - paraphrasable par au cas où la Lorpailleur est folle, je n'y peux rien - et une lecture interrogative avec une glose comme Je n'y peux rien au fait que la Lorpailleur soit folle (ou non). Les deux lectures ne peuvent pas être démêlées, ce qui à notre sens, atteste de la porosité de la frontière entre régimes et circonstants en si.

Le même phénomène de métanalyse est semble-t-il attesté entre le si interrogatif et le si circonstanciel de l'espagnol [Montolío-Durán, 1999 : 3717]. En anglais, les morphèmes ne sont pas homonymes comme en français et en espagnol, mais il a été observé que le morphème if a tendance à supplanter whether dans l'usage quotidien. Ainsi, en colloquial English, on peut employer if pour whether [Renchon, $1967: 133$ ] : see if / I asked if... Il existe des récits de fiction dans lesquels le relateur if est systématiquement substitué à whether [Duval, 2003 : 295]. Le commentaire suivant de Traugott [1985:304n] va dans le même sens :

\begin{abstract}
It should be noted that expressions like If he comes or not doesn't matter and Whether he comes or not doesn't matter are actually compatible with any world at all, and are not strictly about alternatives. This appears to be a function of the negative in the main clause ; also, in this case, if has been replaced by whether, not vice versa, so that the bleaching of the either-or meaning of whether is not surprising.
\end{abstract}

En espagnol et en anglais, on observe donc un phénomène semblable d'indifférenciation entre les si $P$ « régime » et les si $P$ « circonstant». 


\subsection{Métanalyse circonstant - énonciation autonome}

Il faut également composer avec certaines suites ambiguës qui neutralisent l'opposition entre microsyntaxe (les circonstants) et macro-syntaxe (les énonciations autonomes).

Les exemples (22) hésitent entre les deux ordres de combinatoire :

(a) $\mathrm{Si}$ tu dois fermer les fenêtres par où tu regardes, ferme plutôt les yeux. [Porchia, Voix]

(b) $S i$ la barre d'affichage est allumée, observer l'indication. [distributeur de café]

L'élément Q de (22) a une valeur d'ordre ou de recommandation. Deux analyses radicalement différentes sont possibles, sans qu'une nuance sémantique soit aisément décelable :

1) Ou l'hypothèse porte sur le contenu de $Q$. Dans ce cas, le membre si $P$ fait partie du même acte d'énonciation. La structure - micro-syntaxique - est paraphrasable par : je t'ordonne (que) [si $P$, $Q]$. L'élément si $P$ ouvre un espace de validité pour les opérations sémantiques réalisées dans $Q$.

2) Ou l'hypothèse porte sur l'énonciation de Q. Dans ce cas, la structure est paraphrasable par : si P [je t'ordonne (que) Q]. Dans cette lecture macro-syntaxique, $\mathrm{P}$ et $\mathrm{Q}$ sont deux actes d'énonciation distincts. L'élément si $P$ ouvre un espace pour donner un ordre.

La différence que fait Van der Auwera [1986 : 198-199] entre « conditional speech acts » et « speech acts about conditionals » est à notre sens une conséquence de cette métanalyse et coïncide assez bien avec nos analyses. La modalité peut porter sur l'ensemble de la structure ou uniquement sur la valeur « illocutoire » de Q.

De notre point de vue, il est fondamental de ne pas conclure à un continuum des régimes aux énonciations autonomes en passant par les circonstants. Il y a bien, pour les structures en si $P$, trois statuts formels différents. Les métanalyses démontrent qu'il y a des négociations quant à l'endroit où passe la frontière entre ces formats syntaxiques.

\section{Récapitulatif : le profilage informationnel des éléments si $P$}

Au terme de cette étude, on peut proposer une topographie des énoncés en $s i$ du français contemporain en fonction de l'interface syntaxe - stratification informationnelle (cf. tableau, infra).

On notera d'une part que lorsque l'élément si $P$ est intégré ( $V$ si $P$ ), l'interprétation <énonciation autonome> est exclue. D'autre part, seuls les si $P$ circonstants et les énonciations autonomes conduisent à la valeur référentielle d'hypothèse.

Dans le tableau ci-dessous, les cases grisées représentent les cas de figure non attestés.

\begin{tabular}{|c|c|c|c|c|c|}
\hline & $\begin{array}{l}\text { si } P \text { est un } \\
\text { REGIME } \\
\text { (argument } \\
\text { du verbe) }\end{array}$ & $\begin{array}{l}\text { métanalyse } \\
\text { régime - } \\
\text { circonstant }\end{array}$ & $\begin{array}{l}\text { si } P \quad \text { est un } \\
\text { CIRCONSTANT } \\
\text { (adjoint au verbe) }\end{array}$ & $\begin{array}{l}\text { métanalyse } \\
\text { circonstant - } \\
\text { énonciation } \\
\text { autonome }\end{array}$ & $\begin{array}{l}\text { si } P \text { est une } \\
\text { ENONCIATON } \\
\text { AUTONOME }\end{array}$ \\
\hline $\begin{array}{l}\text { L'ELEMENT } \\
\text { SI P EST } \\
\text { DETACHE }\end{array}$ & $\begin{array}{l}\text { (3) antéposé } \\
{[+ \text { substrat }]} \\
\text { (4) postposé } \\
{[+ \text { substrat }]}\end{array}$ & (21) $[-$ foyer $]$ & $\begin{array}{l}\text { (5) antéposé }[- \text { foyer }] \\
(12) \text { cas particulier } \\
{[+ \text { foyer }]} \\
\text { (6) postposé }[- \text { foyer }]\end{array}$ & (22) $[-$ foyer $]$ & $\begin{array}{l}(17)-(20) \\
{[- \text { foyer }]}\end{array}$ \\
\hline $\begin{array}{l}\text { L'ELEMENT } \\
\text { SI P EST } \\
\text { INTEGRE }\end{array}$ & $\begin{array}{l}(1) \\
{[- \text { substrat }]}\end{array}$ & & (7)-(11) [+foyer $]$ & & \\
\hline
\end{tabular}

Tableau récapitulatif 
Nous avons pris en compte au moins trois facteurs pour établir les correspondances entre le plan syntaxique et le plan informationnel : (i) le rapport syntaxique entre si $P$ et $\mathrm{Q}$, (ii) la position de si $P$ (antéposé ou postposé) et (iii) l'intégration à l'intérieur du prédicat. Les signifiants prosodiques qui affectent si $P$ jouent également un rôle non négligeable. On a tenté de montrer comment la structure informationnelle est marquée par la syntaxe, dans les cas où des indices formels permettent de prendre une décision ${ }^{18}$.

Résumons.

(1) Dans les si $P$ régimes, le détachement est un moyen de substratifier si $P$ (cf. le phénomène de doublemarquage : le complément est réalisé à la fois lexicalement et pronominalement). Dans sa position non marquée (intégrée à droite), le constituant si $P$ endosse le statut de foyer par défaut.

(2) Pour les circonstants si $P$, c'est l'intégration à l'intérieur du prédicat qui est l'opération marquée : cette procédure revient à focaliser le circonstant. En position extra-prédicative (non marquée), si $P$ est le substrat par défaut.

Par ailleurs, il convient de tenir compte des contextes d'enchâssement et de la longueur du segment si $P$ : ces contraintes sont partiellement indépendantes du marquage de la stratification informationnelle. Dans (12), le constituant si $P$ est placé en tête de structure et focalisé : c'est l'intégration prédicationnelle qui est ici l'indice déterminant pour la focalisation. Ces aspects nous conduisent à parler de correspondances plutôt que d'isomorphisme entre la syntaxe et la structure informationnelle ${ }^{19}$.

(3) Pour les énonciations si $P$, la position et le détachement (elles sont toujours détachées) ne constituent pas des indices concluants pour marquer la structure informationnelle. A notre sens, la position marque plutôt l'ordonnancement des opérations praxéologiques.

\section{Références bibliographiques}

Austin, J. L. (1961 ${ }^{3}$ ). Ifs and Cans. Philosophical papers. Oxford : Oxford University Press, 205-232.

Berrendonner, A. (2002a). Les deux syntaxes. Verbum, XXIV, 23-35.

Berrendonner, A. (2002b). Morpho-syntaxe, pragma-syntaxe et ambivalences sémantiques. In Andersen H. L. \& Nølke H. (ed), Macro-syntaxe et macro-sémantique. Berne : P. Lang, 23-41.

Blanche-Benveniste, C. (1981). La complémentation verbale : valence, rection, associés. Recherches sur le français parlé, 3, 57-98.

Blanche-Benveniste, C. \& al. (1991). Le français parlé : études grammaticales. Paris : éd. du CNRS.

Blinkenberg A. (1950). Le problème de l'accord en français moderne. Essai d'une typologie. Copenhague : E. Munksgaard.

Butler, C. S. (2005). Focusing on focus : A comparison of Functional Grammar, Role and Reference Grammar and Systemic Functional Grammar. Language Sciences, 27-6, 585-618.

Corminboeuf, G. (à paraître, a). L'antéposition des interrogatives indirectes totales en français contemporain. In : Les linguistiques du détachement, Actes du colloque de Nancy, 2006.

Corminboeuf, G. (à paraître, b). L'expression de l'hypothèse en français contemporain, entre hypotaxe et parataxe, thèse de doctorat, Neuchâtel.

Cornulier de, B. (1985). Effets de sens. Paris : Minuit.

Dancygier, B. \& E. Sweetser (2005). Mental Spaces in Grammar : Conditional constructions. Cambridge : CUP.

Delaveau, A. (1980). Questions sur l'analyse de 'si'. Linx, 2, 7-34.

Ducrot, O. (1984). Le dire et le dit. Paris : Minuit.

Duval, M. (2003). Le problème de l'interrogation indirecte totale d'après ses marques (en français, anglais et coréen). Étude contrastive et typologique. Thèse de doctorat Paris IV - Sorbonne. 
Ford, C. \& Thompson S. (1986). Conditionals in discourse : A Text-based study from English. In : E. Traugott \& al. (éd), On Conditionals. Cambridge : CUP, 353-372.

Haiman, J. (1978). Conditionals are topics. Languages, 54/3, 564-589.

Hamon, S. (2002). Les conjonctions causales et la propriété d'enchâssement. LINX, 46, 25-36.

Kronning, H. (1996). Modalité, cognition et polysémie : sémantique du verbe modal 'devoir. Uppsala / Stockholm : Acta Universitatis Upsaliensis.

Lacheret-Dujour, A. (2003). La prosodie des circonstants en français parlé. Leuven-Paris : Peeters.

Lambrecht, K. (1994). Information structure and sentence form : topic, focus, and the mental representations of discourse referents. Cambridge : CUP.

Montolío Durán, E. (1999). Las construcciones condicionales. In Bosque I. \& V. Demonte (ed), Gramática descriptiva de la lengua española. Real Academia Española. t. III. Madrid : Espasa, 3643-3737.

Nølke H. (1994). Linguistique modulaire : de la forme au sens. Louvain-Paris : Peeters.

Renchon, H. (1967). Etudes de syntaxe descriptive, t. 1. Bruxelles : Palais des Académies.

Rossi, M. (1999). L'intonation, le système du français : description et modélisation. Paris : Ophrys.

Rothenberg, M. (1989). Quelques moyens syntaxiques de rhématisation et de thématisation en français. Bulletin de la société de linguistique de Paris, LXXXIV, 143-161.

Sandfeld, K. (1977). Syntaxe du français contemporain. Les propositions subordonnées. Genève : Droz.

Touratier, C. (1993). Structure informative et structure syntaxique. BSL, 88.1, 49-63.

Touratier, C. (1994). Syntaxe latine. Louvain-la-Neuve : Peeters.

Traugott, E. C. (1985). Conditional markers. In Haiman J. (ed), Iconicity in Syntax. Amsterdam / Philadelphia : Benjamins, 289-307.

Van der Auwera, J. (1986). Conditionals and speech acts. In Traugott E. C. \& al. (ed), On Conditionals. Cambridge : CUP, 197-214.

Vogüé de, S. (1986). La conjonction si et la question de l'homonymie. BULAG, 13, 105-189.

\footnotetext{
${ }^{1}$ Les « propositions complexes » sont peu étudiées dans cette perspective.

${ }^{2}$ Les signifiants prosodiques apportent également des indices pertinents pour établir les correspondances entre ces deux plans de l'analyse linguistique.

${ }^{3}$ Le travail de Butler [2005], qui compare trois théories fonctionnelles - la Fonctional Grammar, la Role and Reference Grammar et la Systemic Functional Grammar -, démontre les difficultés liées à une telle entreprise.

${ }^{4}$ Il conviendrait d'éviter d'identifier « information nouvelle» et «pertinence de l'information ». De l'information « ancienne » peut être pertinente, ou du moins présentée comme telle [Butler, $2005: 612-613$ ].

${ }^{5}$ On peut se demander s'il y a un seul si hypothétique qui endosse des effets de sens en contexte ou plusieurs $s i$ homonymiques; on se ralliera à la première solution (pour une discussion, de Cornulier [1985] et de Vogüé [1986]).

${ }^{6}$ Dans l'exemple (2a), on observe deux unités graphiques (auxquelles pourraient correspondre, à l'oral, deux unités prosodiques). Dans les exemples (4) infra, l'élément si $P$ est détaché à droite, mais au sein de la même unité graphique (la même unité prosodique à l'oral). On différenciera les « epexégèses » (2a) des détachements à droite (4).

${ }^{7} \mathrm{Au}$ plan sémantique, des distinctions peuvent être réalisées en fonction du profil épistémique de l'opérateur d'ouverture d'univers si $P$. Le contenu de $P$ peut se présenter épistémiquement comme conforme à la réalité, itératif, contingent ou contrefactuel. On n'entrera pas ici dans ces considérations sémantiques.

${ }^{8}$ Le terme «postfixe » renvoie à une indication prosodique : le postfixe a un contour «plat», un ton bas, non accentué [Blanche-Benveniste \& al., $1991: 166]$.

${ }^{9}$ On peut se reporter à l'étude de Lacheret [2003] pour des exemples de circonstants postposés avec une intonation de « parenthèse ». La position finale de période ne coïncide pas forcément avec le foyer.
} 
${ }^{10}$ Pour Rossi [1999 : 115], « Le morphème intonatif conclusif CC est l'opérateur de rhématisation. [...] Le foyer est constitué des éléments syntaxiques qui demeurent devant le morphème intonatif conclusif majeur CC ».

11 Selon Montolío-Durán [1999 : 3705], c'est ce statut de foyer, dans des exemples de ce genre, qui expliquerait l'impossibilité d'introduire la particule alors à l'initiale de Q. Montolío-Durán présente l'exemple suivant, semble-t-il plus naturel en espagnol qu'en français : Sólo si hace sol, iré a la playa. De même en anglais, chez Dancygier \& Sweetser [2006 : 225] : Only if you pay me \$20 will I mow the lawn.

${ }^{12}$ Dans (13), les parenthèses angulaires démarquent l'opérande.

${ }^{13}$ L'explication syntaxique proposée est congruente avec ce qui était visé par les paraphrases au moyen de verba dicendi de Sandfeld [1965 : 344-345] et Ducrot [1984 : 108] : si tu as soif, je t'informe de ce qui suit : il y a de la bière dans le frigo.

${ }^{14}$ La notion de clause utilisée ici est à distinguer de son homonyme en linguistique anglo-saxonne. Quant à l'énonciation, elle n'a rien à voir avec un « acte illocutoire ».

${ }^{15}$ Dans la partie si $P$ de la majorité des exemples, il n'y a pas d'information cataphorique à proprement parler : c'est quand on considère la structure en entier, au terme de l'interprétation, que l'on peut parler de cataphore, de manière rétroactive. La cataphore est cependant verbalisée dans des exemples comme : Si nous y réfléchissons, Figaro a le même aspect nonchalant, et en pleine fronde, Don Sanche aurait pu jouer le rôle de Figaro [Brasillach < Frantext]. Le premier terme de la cataphore est le pronom $y$, alors que le second est ellipsé. Le rapport cataphorique ne s'opère pas entre $\mathrm{P}$ et $\mathrm{Q}$, mais entre deux constituants de $\mathrm{P}$, dont l'un est ellipsé.

${ }^{16}$ L'élément pourrait être interprété également par rapport au fait nommé dans l'énonciation qui suit : <à propos d'une voiture> Si ça se trouve elle est lavée à l'œeil par des troufions [presse] : ce qui peut «se trouver », c'est le fait même que Q [de Cornulier, 1985 : 62].

${ }^{17}$ Il conviendrait de distinguer deux types de substrat : 1) Mis en discours dans le cadre d'un " coup énonciatif» unique, les compléments si $P$ (régimes et circonstants) entretiennent un rapport sémantique avec Q. 2) Les versions macro-syntaxiques (§ 4.) sont des constructions à deux coups énonciatifs dans lesquelles les membres entretiennent un rapport de nature pragmatique. Il conviendrait peut-être de conserver le concept de substrat pour ce qui concerne l'organisation intra-clausale 1), et d'utiliser une autre terminologie pour les relations inter-énonciatives 2).

${ }^{18}$ Lorsque si $P$ est un membre parmi d'autres au sein d'une longue période, il est difficile de savoir quel rôle il endosse dans la structuration informationnelle. Exemple : mais je pense qu'il peut apprendre à mieux connaître son entreprise / et à mieux l'organiser pour baisser leurs marges / pour vendre du lait / si ils veulent meilleur marché / ça c'est leur job / hein_c'est la concurrence / c'est leur travail \ [oral radio, débat sur le prix du lait]

${ }^{19}$ Touratier [1993: 62] conclut également, à propos d'autres faits de langue, à une « absence d'isomorphisme » entre les deux structurations. 